POPULATIONS

VULNERABLES $\quad 5 \mid 2019$

Dimensions territoriales et vulnérabilités

\title{
La désindustrialisation comme vecteur de vulnérabilité territoriale
}

Deindustrialization as a vector of territorial vulnerability

\section{Thomas Venet}

\section{OpenEdition}

1 Journals

Édition électronique

URL : https://journals.openedition.org/popvuln/1044

DOI : $10.4000 /$ popvuln.1044

ISSN : 2650-7684

Éditeur

LIR3S - Laboratoire Interdisciplinaire de Recherche "Sociétés Sensibilités Soin" (UMR 7366 CNRS-uB)

Édition imprimée

Date de publication : 1 mars 2019

Pagination : 97-128

ISBN : 978-2-918173-26-7

ISSN : 2269-0182

Référence électronique

Thomas Venet, «La désindustrialisation comme vecteur de vulnérabilité territoriale », Populations vulnérables [En ligne], 5 | 2019, mis en ligne le 26 mai 2020, consulté le 26 février 2022. URL : http:// journals.openedition.org/popvuln/1044; DOI : https://doi.org/10.4000/popvuln.1044

Ce document a été généré automatiquement le 26 février 2022.

Les contenus de la revue Populations vulnérables sont mis à disposition selon les termes de la Licence Creative Commons Attribution 4.0 International 


\title{
La désindustrialisation comme vecteur de vulnérabilité territoriale
}

\author{
Deindustrialization as a vector of territorial vulnerability
}

\author{
Thomas Venet
}

\section{Introduction}

1 Depuis les années 1980, la morphologie sociale de la France a suivi une tendance marquée par la baisse de la part des ouvriers dans la population active et par l'augmentation de la proportion des professions intermédiaires, des cadres, des employés et des chômeurs. La mécanisation de l'industrie, qui demande l'embauche d'une main d'œuvre moins nombreuse mais plus qualifiée, la financiarisation de l'économie et la mondialisation des productions, notamment, se traduisent par une forte baisse du nombre d'ouvriers et de leur part dans la population active en France.

2 Selon les recensements de la population de 1968 et de 2013, la part des ouvriers dans la population active de France métropolitaine est passée de 33,8\% à 18,5\% sur cette période (Figure 1). En parallèle, les cadres, les professions intermédiaires et les employés, symboles de la tertiarisation de l'économie ont vu leurs parts respectives augmenter. En 1968, les cadres représentaient environ $6 \%$ de la population active métropolitaine. Cette proportion atteint $16 \%$ en 2013. Sur la même période, le taux d'employés est passé de $20 \%$ à $24 \%$ de la population active, et les professions intermédiaires de $14 \%$ à $24 \%$. Mais c'est également au cours de cette période que le chômage va s'étendre et s'établir comme une nouvelle composante à part entière du monde du travail. Toujours selon les recensements de 1968 et $2013^{1}$, la part des chômeurs dans la population active est passée de $2,6 \%$ à 10,5\%. Cette augmentation a surtout eu lieu au cours des années 1980 et 1990, et oscille autour de $10 \%$ depuis le début des années 2000 . 
Figure 1. Évolution des parts des Professions et catégories socioprofessionnelles (PCS) dans la population active de France métropolitaine entre 1968 et 2013

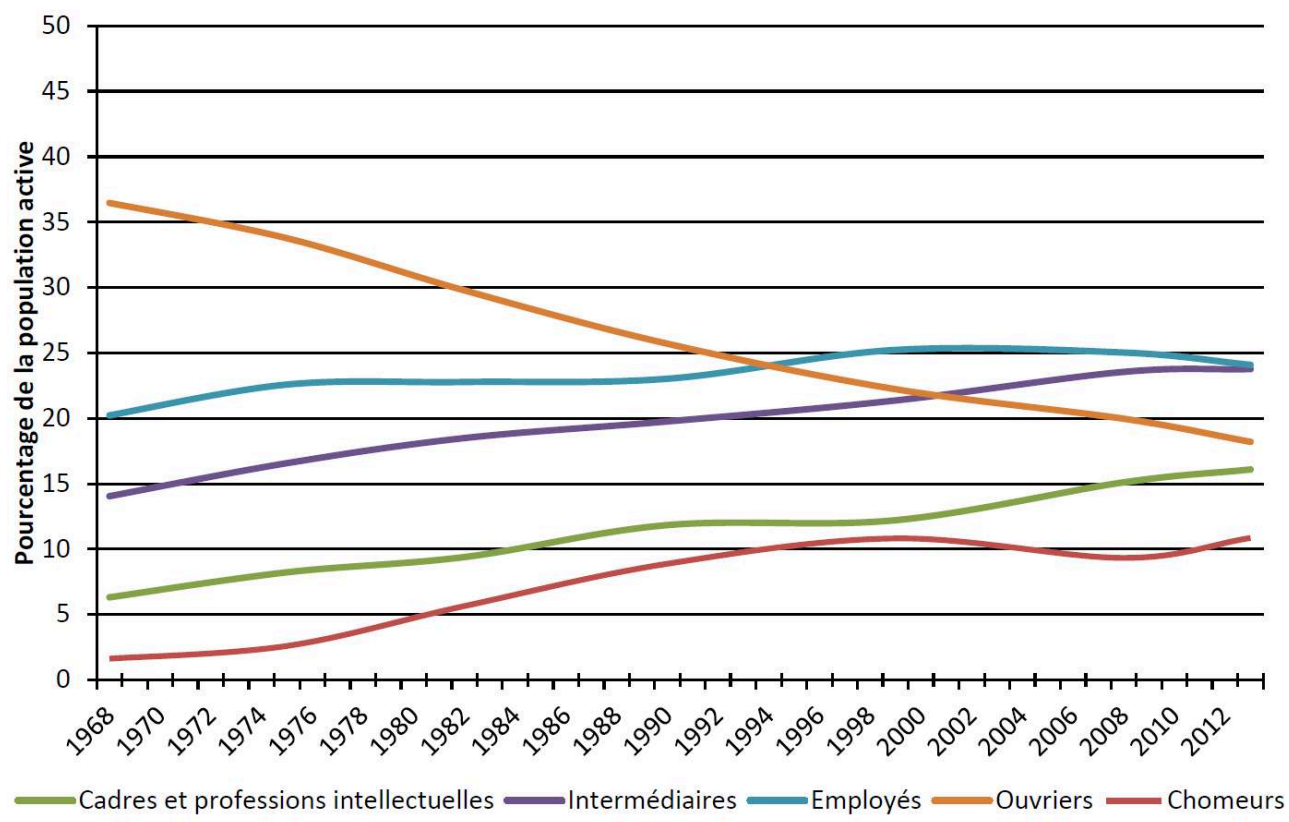

Champs : population active de 25 à 55 ans, France métropolitaine

Sources : recensements de la population de 1968, 1975, 1982, 1990, 1999, 2008 et 2013, INSEE

3 La baisse des effectifs industriels et l'apparition d'un chômage persistant n'ont pas été des phénomènes conjoncturels liés aux difficultés dues à une crise, mais sont le fruit de vastes bouleversements de l'organisation productive (Castel, 2009). Les grandes entreprises fusionnent, mondialisent leurs productions et la rentabilité financière devient l'objectif prioritaire, entraînant la réduction des coûts liés aux matières premières et le « prix » de la main d'œuvre. Dans ce cadre, l'augmentation du nombre de chômeurs et le fort développement du travail temporaire, qui intervient en parallèle d'une réorganisation à flux tendus des productions, vont permettre de composer un volant de main d'œuvre disponible en cas de forte demande (Glaymann, 2005). Cette "flexibilisation» des postes, que l'on peut également interpréter comme un vaste processus de «déstabilisation des stables » (Castel, 1995), génère de nouvelles figures de la pauvreté en modifiant profondément les réalités sociales et économiques de certaines populations. Pour en rendre compte, de nombreux travaux de la sociologie contemporaine se sont focalisés sur les trajectoires des femmes et des hommes qui subissent ces transformations. Par ces travaux, la lumière est portée notamment sur les effets de celles-ci en ce qui concerne les mobilisations collectives (Collectif du 9 août, 2017), les trajectoires professionnelles (Roupnel-Fuentes, 2011), ou encore les mobilités géographiques et recompositions familiales (Vignal, 2005).

Promues dans les années 1980, les théories qui analysaient les transformations sociales comme un processus de "moyennisation " généralisée de la société (Mendras, 1988) sont alors profondément remises en cause, et laissent apparaitre de profonds clivages entre les territoires. C'est ce que montrait Laurent Davezies (2012) dans un effort pour "déglobaliser la crise» de 2008-2009. Ainsi, au niveau français, on observe que les pertes d'emplois sont principalement situées dans le Nord du pays (dans les régions les plus industrielles) et ont très peu affecté les régions du Sud et l'Ouest (constituant des zones plus touristiques). C'est dans les grandes villes, où le secteur tertiaire est 
fortement développé, que l'emploi a été le moins affecté alors que les espaces les plus touchés sont ceux qui étaient déjà en difficulté avant la crise, c'est-à-dire les territoires ruraux et industriels éloignés des métropoles. Au final, "la crise a attaqué prioritairement les territoires déjà blessés » (p. 33), déstabilisant surtout les ouvriers qui constituent une grande partie de la population de l'espace rural.

Nous nous intéresserons ici spécifiquement au phénomène de désindustrialisation en Picardie (regroupant les départements de la Somme, de l'Aisne et de l'Oise) et à ses effets sur les territoires qui ont été les plus affectés. Cette région est celle qui compte la plus forte proportion d'ouvriers dans sa population active à la fin des années 1960 ( $47,3 \%$ en 1968), devant l'Alsace (44,5\%), la Lorraine (42,8 \%) et l'île-de-France (43,5\%). Depuis cette période, on observe une chute rapide et continue de la part des ouvriers dans la population active régionale (Figure 2). Selon le recensement de 2013, les ouvriers représentent environ $24 \%$ de la population active de la Picardie, ce qui reste supérieur à la moyenne nationale $(18,5 \%)$.

6 Les parts des cadres, professions intellectuelles et intermédiaires et des employés au sein de la population active picarde ont quant à elles augmenté, mais dans une moindre mesure si l'on compare avec les évolutions nationales. En 2013, 11 \% de la population active picarde occupe un emploi de cadre ou profession intellectuelle, $23 \%$ occupe une profession intermédiaire, $25 \%$ est employé.

Figure 2. Évolution des parts des PCS dans la population active de Picardie depuis 1968

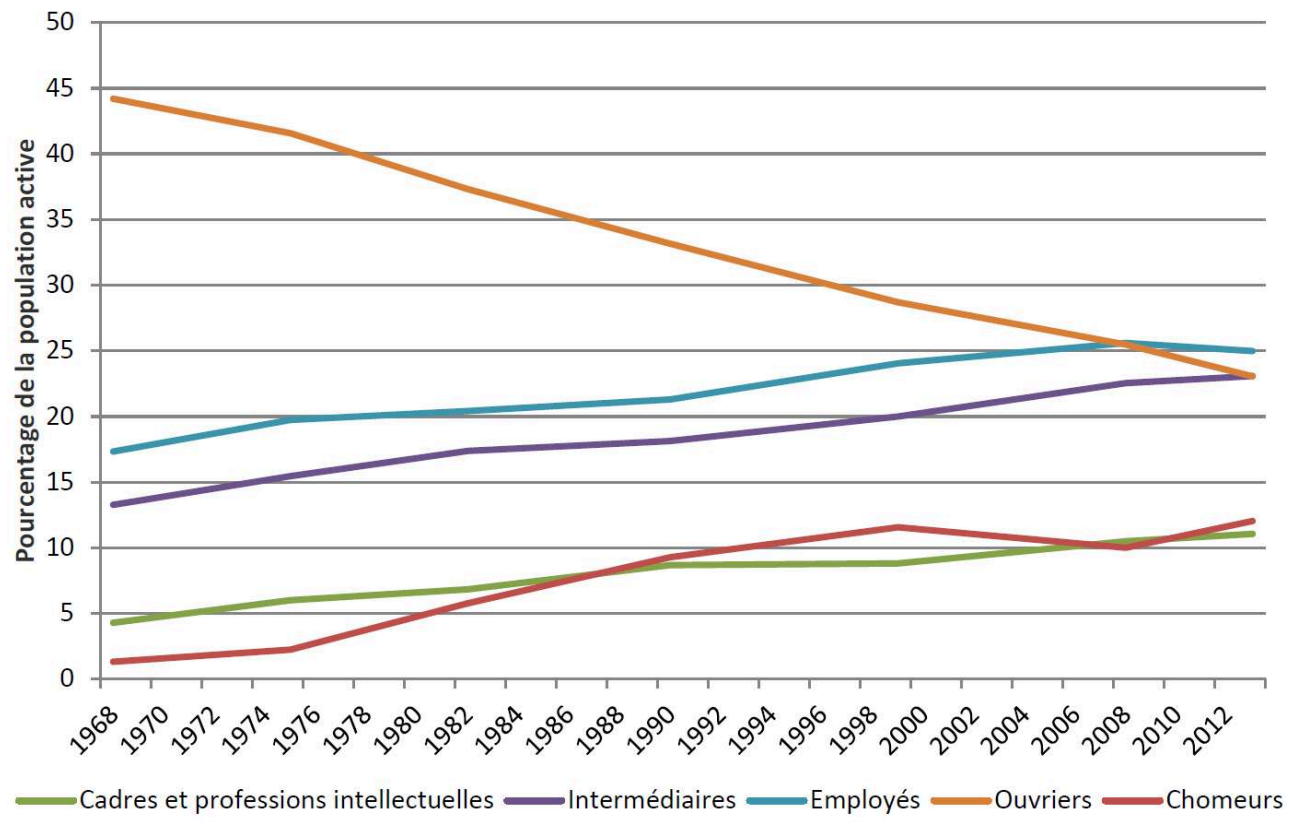

Champs : population active de 25 à 55 ans, Picardie. Afın d'alléger la lecture du graphique, les PCS Agriculteurs et Artisans-Commerçants ont été retirées

Sources : recensements de la population de 1968, 1975, 1982, 1990, 1999, 2008 et 2013, INSEE

7 En parallèle, le taux de chômage a connu une augmentation plus importante en Picardie qu'en moyenne nationale. Le taux de chômage picard était inférieur au taux national avant 1982, mais a connu ensuite une augmentation continue jusqu'en 1999. À l'échelle nationale comme régionale, le recensement de 2007 témoigne d'un recul du taux de chômage qui est pourtant reparti à la hausse en 2013. À cette date, la part des 
chômeurs dans la population active picarde dépasse les $12 \%$, alors qu'elle était inférieure à 1,5\% en 1968.

8 Outre son caractère industriel, la Picardie est une région à dominante rurale. En 2012, 94,5\% des communes de Picardie sont classées parmi les zones à dominantes rurales, contre $87,6 \%$ des communes françaises (Figure 3 ). Très peu de communes picardes composent de grands pôles urbains ( $3 \%$ contre presque $9 \%$ au niveau national). En revanche, les zones qui regroupent le plus les populations rurales (c'est-à-dire les communes appartenant aux couronnes des petits pôles, les autres communes multipolarisées et les communes isolées) sont surreprésentées.

Les caractères ruraux et industriels de la Picardie ne sont pas contradictoires. En effet, les ouvriers représentent une part importante de la population des communes rurales ${ }^{2}$. Ce caractère populaire des mondes ruraux est particulièrement perceptible en Picardie, où l'industrie s'est développée sous forme de sites industriels s'implantant principalement à la campagne. Différents secteurs (textile, sucreries, petites métallurgies) s'installent très tôt en Picardie et font travailler des ouvriers-paysans, cultivant la terre et travaillant une partie du temps pour un patron, le plus souvent pour une rémunération « à la pièce » (Noiriel, 2002).

Figure 3. Zonage en aires urbaines et en aires d'emploi de l'espace rural (ZAUER) de la Picardie

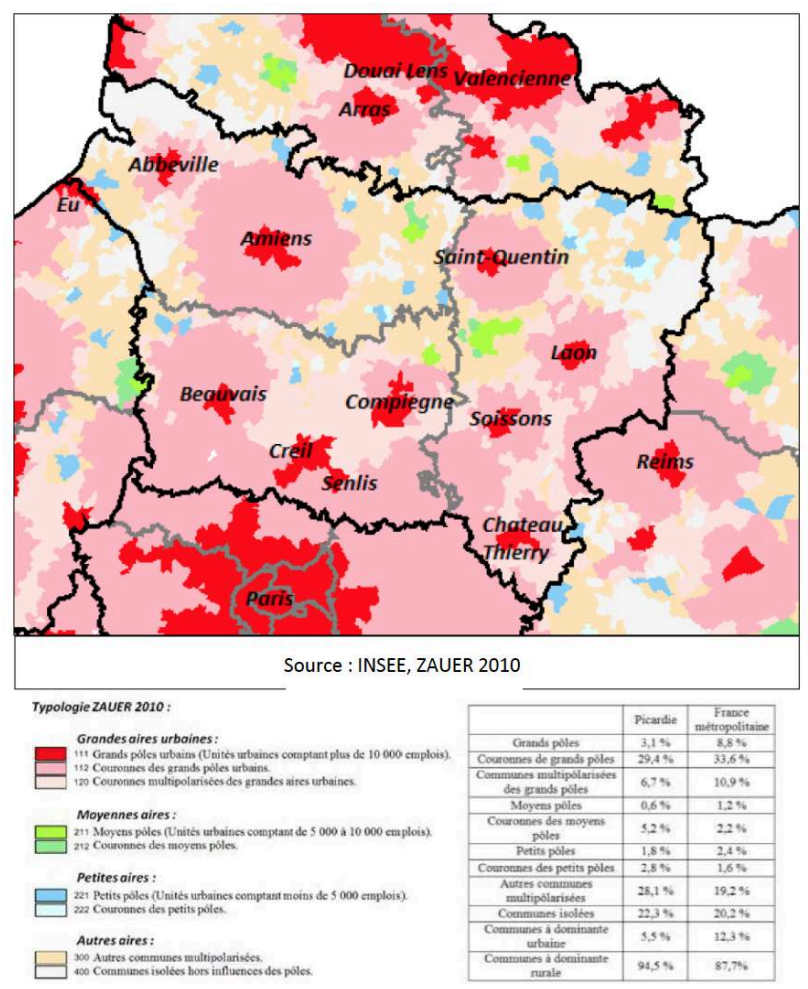

10 L'industrie, en se développant, a fortement affecté les modes de vie et l'on peut considérer que le mouvement de désindustrialisation amène lui aussi un ensemble de bouleversements qui affectent ces territoires. L'apparition du chômage de masse n'est pas la seule manifestation des difficultés éprouvées par les populations sur les territoires désindustrialisés, et leurs situations peuvent être perçues comme des formes de vulnérabilité particulières (Castel, 1994). 
11 Pour tenter de mettre en évidence les effets concrets induits par la désindustrialisation au niveau des territoires, il faudra analyser conjointement des dynamismes sociaux, économiques et démographiques situés à des échelles géographiques fines. Appréhender la vulnérabilité des territoires, c'est prendre en compte différents niveaux d'information. En elle-même, la vulnérabilité est à analyser sous le prisme des inégalités sociales (de revenu, de santé ou encore de formation) et de leur dynamisme afin d'éviter les discours tendant vers un potentiel empowerment des acteurs ${ }^{3}$. Cette vulnérabilité territoriale ne peut également être considérée comme figée, elle est directement liée aux transformations socio-économiques affectant les territoires en continu. Enfin, elle doit être analysée de manière relative en portant le regard sur les évolutions concernant les « vulnérables », mais également les « protégés ».

12 Afin de mener cette analyse des vulnérabilités territoriales, nous nous sommes appuyé sur une base de données construite par agrégation d'indicateurs issus de différentes sources (Recensement, Fichier localisé social et fiscal, données de Pôle Emploi, des Impôts) à l'échelle des communes et des cantons. Cette base de données nous permettra de dresser un portrait des territoires qui composent la région Picardie, en nous basant sur des variables relatives aux évolutions et aux structures de l'emploi, à la morphologie sociale. Ces variables pourront ensuite être mises en lien avec différents indicateurs renvoyant à des situations de vulnérabilité économique ou démographique.

Dans un premier temps, nous utiliserons ces indicateurs pour décrire finement les transformations de l'emploi et de la morphologie sociale de Picardie, en portant une attention particulière à la localisation de celles-ci. Nous construirons ensuite une analyse en composantes principales (ACP) permettant de traiter ensemble les variables de transformation de l'emploi et de la morphologie sociale, qui pourrons alors être mises en lien avec d'autres variables (pauvreté et revenus, dynamismes et structure démographiques) renvoyant aux situations de vulnérabilité des populations considérées.

\section{Les transformations de l'emploi et de la morphologie sociale en Picardie}

Entre 1982 et 2013, le nombre d'emplois au lieu de travail a augmenté de 20,1\% en Picardie, contre $26,9 \%$ en France métropolitaine. Ce dynamisme, globalement plus faible que la moyenne nationale, n'est toutefois pas homogène au sein de la région. Ainsi, le nombre d'emplois a connu une plus forte augmentation dans l'Oise $(32,7 \%)$ que dans la Somme (15\%) et l'Aisne (7,7 \%) (Tableau 1).

Tableau 1. Taux d'évolution (en \%) du nombre d'emplois entre 1982 et 2013

\begin{tabular}{|l|l|l|l|l|l|}
\hline & Emploi total & Agriculture & Industrie & BTP & Services \\
\hline Picardie & 20,1 & $-57,3$ & $-44,0$ & 11,3 & 76,0 \\
\hline Aisne & 7,7 & $-52,2$ & $-49,8$ & 19,7 & 56,2 \\
\hline Oise & 32,7 & $-60,0$ & $-44,3$ & 12,0 & 99,2 \\
\hline
\end{tabular}




\begin{tabular}{|l|l|l|l|l|l|}
\hline Somme & 15,0 & $-60,1$ & $-37,3$ & 2,2 & 63,5 \\
\hline France métropolitaine & 26,9 & $-56,2$ & $-36,1$ & 2,1 & 69,9 \\
\hline
\end{tabular}

Champs : emplois au lieu de travail dans les communes de Picardie

Sources : recensements de la population de 1982 et 2013, INSEE

Figure 4. Évolution du nombre d'emplois (tous secteurs confondus) au lieu de travail dans les communes de Picardie entre 1982 et 2013

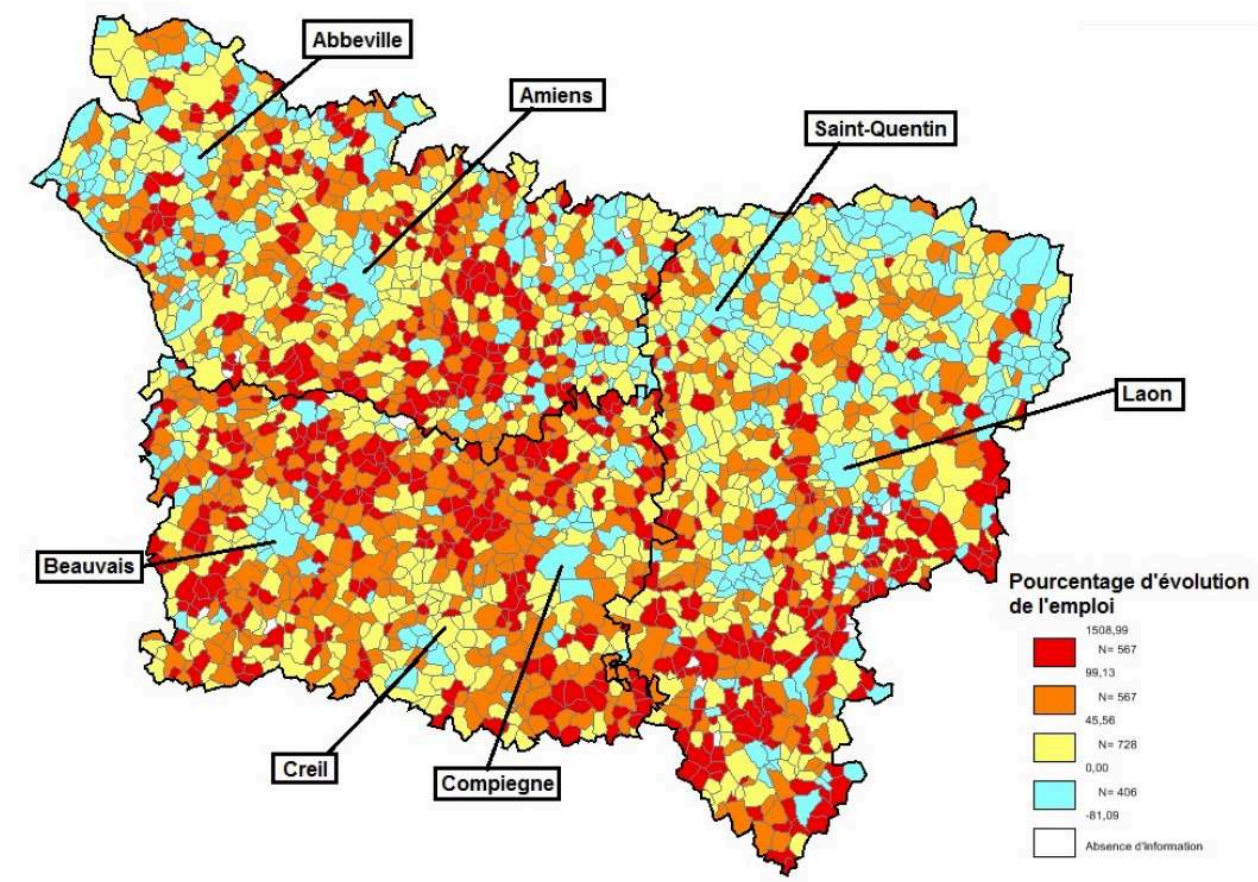

Champs : emplois au lieu de travail dans les communes de Picardie

Sources : recensements de la population de 1982 et 2013, INSEE

À une échelle plus fine encore, durant cette période, certains territoires ont connu une baisse du nombre d'emplois sur place. Les communes rurales sont les plus concernées (Figure 4). Le Nord de l'Aisne et de la Somme concentre la grande majorité des communes dont le nombre d'emplois au lieu de travail a baissé entre 1982 et 2013. L'Oise et le Sud de l'Aisne, plus proches de la région parisienne, regroupent pour leur part les communes où le nombre d'emplois à le plus augmenté. Les centres urbains sont également caractérisés par des dynamiques de l'emploi assez nuancées. Celui-ci a eu tendance à y décroître, tous secteurs confondus, depuis les années 1980.

\section{1) Évolution des emplois par secteurs d'activité en Picardie}

L'évolution de l'emploi en Picardie depuis le début des années 1980 peut être analysée en deux phases. Entre 1982 et 1999, le nombre d'emplois dans la région a fortement augmenté, en Picardie $(+23,4 \%)$ comme en France métropolitaine $(+23 \%)$. Depuis la fin des années 1990, cette progression a ralenti, et le nombre d'emplois en Picardie tend même à reculer à partir de la fin des années 2000. Entre 1999 et 2013, le nombre 
d'emplois en France métropolitaine augmente de 3,1\%, mais baisse de 2,7\% en Picardie.

Dans les années 1980 et 1990, l'emploi a augmenté dans les trois départements de Picardie, mais avec une intensité plus forte dans l'Oise $(+32,8 \%)$ que dans la Somme $(+18,5 \%)$ et l'Aisne $(+15,3 \%)$. Au cours de cette période, au niveau régional, l'emploi dans le secteur des services s'est considérablement développé. Le nombre d'emplois dans ce secteur a augmenté de 61,4\%, alors que l'emploi industriel a décliné de 14,4\%. Ces tendances s'observent avec des intensités légèrement différentes selon les départements. L'Aisne est le département où l'emploi industriel a le plus reculé ($20,9 \%)$ et où l'emploi dans les services s'est le moins développé $(+49,4 \%)$. C'est dans l'Oise que l'augmentation du nombre d'emplois des services a été la plus forte (+77,6\%), et l'emploi industriel y a baissé de 13,2\%. Enfin, dans la Somme, le nombre d'emplois industriels a connu la plus faible baisse de la région (- 9,5\%), et le nombre d'emplois dans les services a augmenté de 50,7\%.

Depuis la fin des années 1990, l'augmentation du nombre d'emplois dans les services a ralenti, alors que les pertes dans le secteur industriel se sont accélérées (Figure 5). Entre 1999 et 2013, l'emploi dans les services a augmenté de $9 \%$ en Picardie (contre $11,4 \%$ en France métropolitaine), alors que l'industrie y a perdu 34,5 \% de ses emplois (contre 26,5\% au niveau national). Ainsi, l'évolution de l'emploi tous secteurs confondus est négative dans les trois départements entre 1999 et 2013 . Dans l'Aisne et la Somme, ces pertes sont plus marquées que dans l'Oise (respectivement - 6,6\%; - $3 \%$ et $-0,1 \%)$. Dans ce dernier département, l'emploi dans les services a connu une augmentation légèrement supérieure aux moyennes régionales et nationales $(+12,1 \%)$, alors que l'Aisne et la Somme sont bien en-deçà (+ 4,6 \% et + 8,5\%). Les pertes d'emplois industriels dépassent pour leur part les $30 \%$ dans les trois départements.

Figure 5. Évolution du nombre d'emplois (pour 100 emplois en 1982) par secteurs d'activités en Picardie

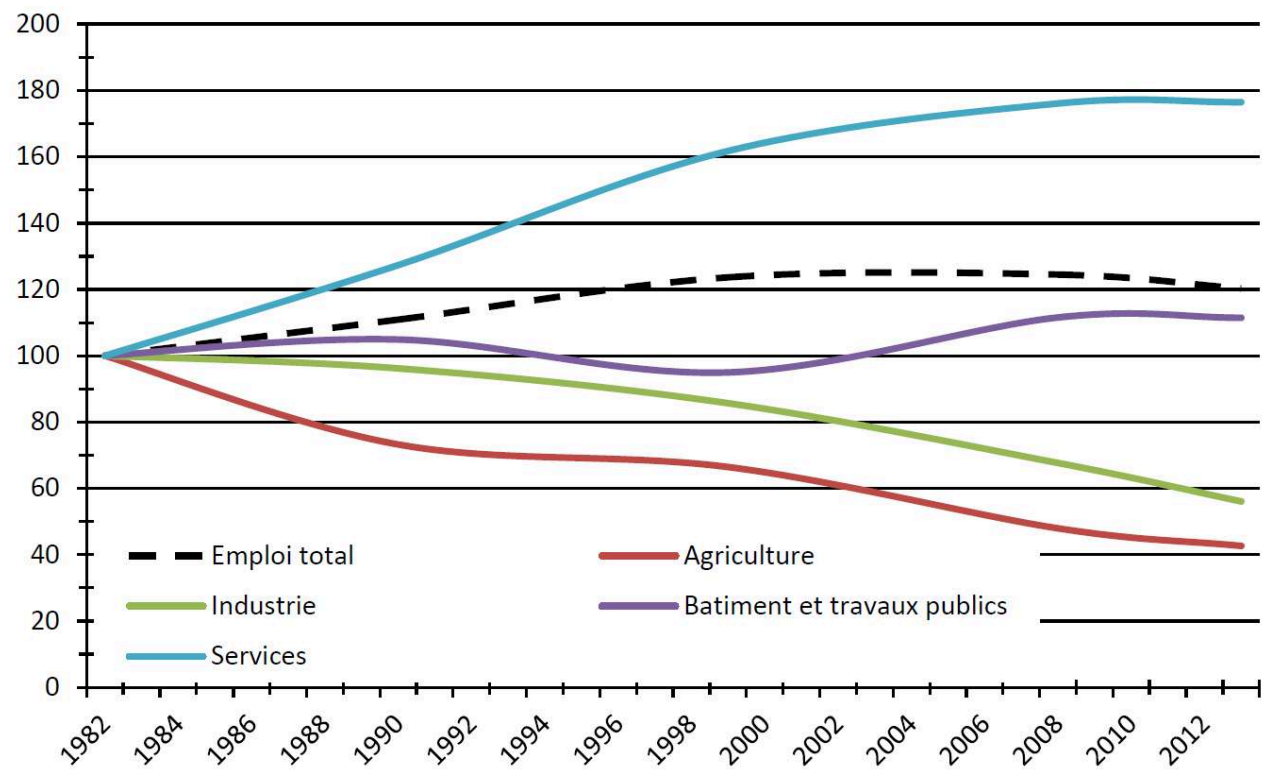

Champs : emplois au lieu de travail en Picardie

Sources : recensements de la population de 1982, 1990, 1999, 2008 et 2013 

l'ensemble de la région, avec des zones de concentration assez marquées dans le Nord de l'Aisne, le Sud de la côte Picarde et les zones frontalières entre le Somme, l'Aisne et l'Oise. En 2013, seules 11,1\% des communes de Picardie atteignent le seuil des $30 \%$ des emplois dans l'industrie. Les zones qui restent les plus industrielles se situent sur la côte Picarde, à la frontière avec la Seine-Maritime et dans le Nord de la Somme et de l'Aisne (Figures 6 et 7).

Figure 6. Les communes de Picardie selon la part des emplois dans l'industrie en 1982

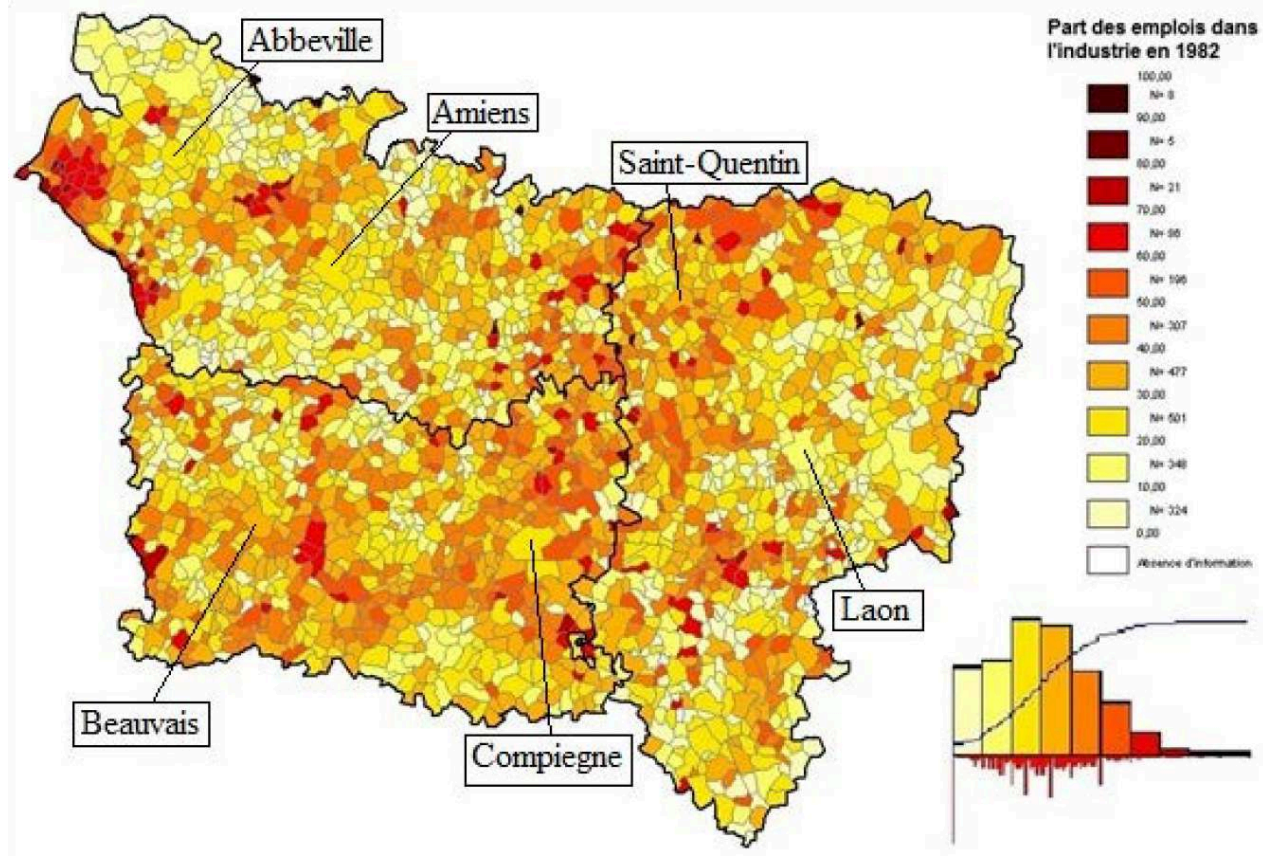

Champs : emplois au lieu de travail dans l'industrie en Picardie

Sources : recensements de la population de 1982 
Figure 7. Les communes de Picardie selon la part des emplois dans l'industrie en 2013
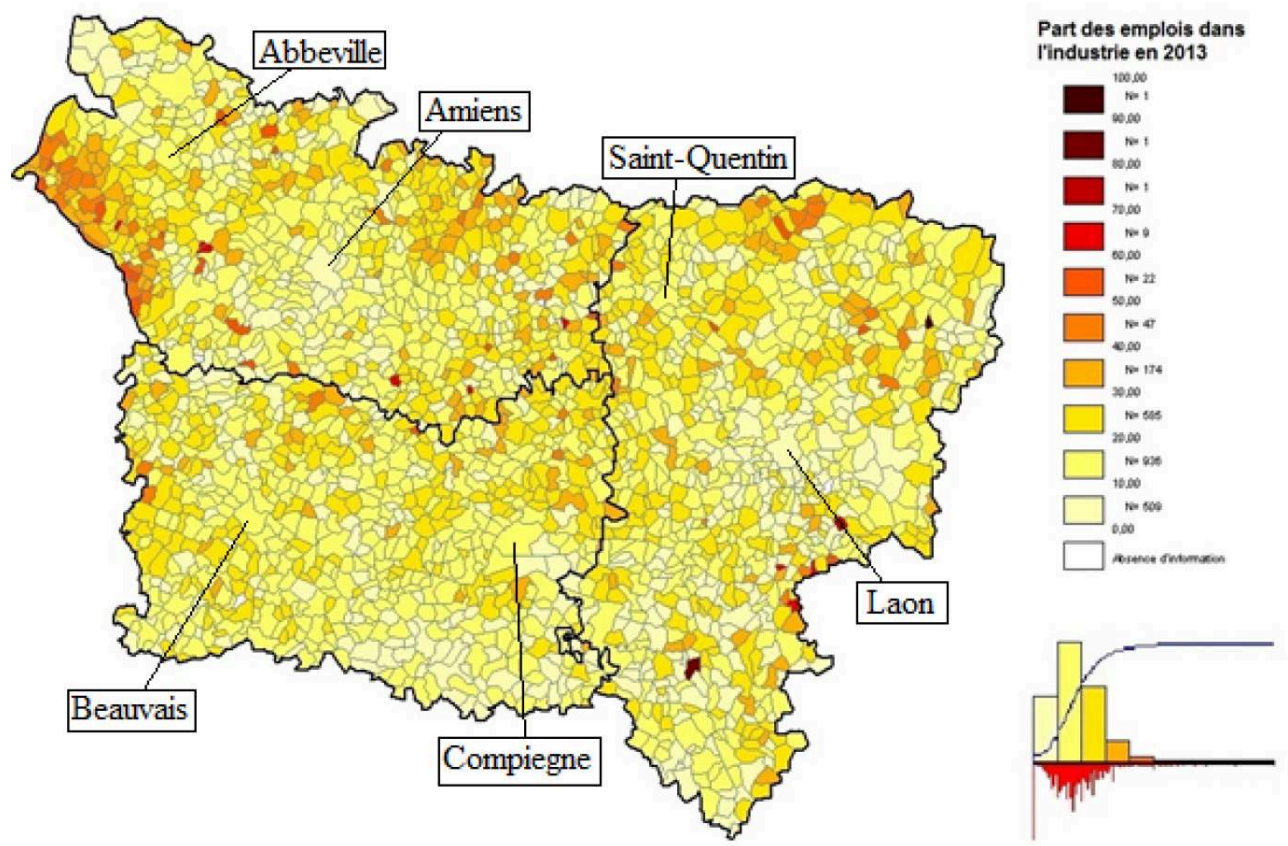

Champs : emplois au lieu de travail dans l'industrie en Picardie

Sources : recensements de la population de 2013

Figure 8. Les communes de Picardie selon la part des emplois dans les services en 1982

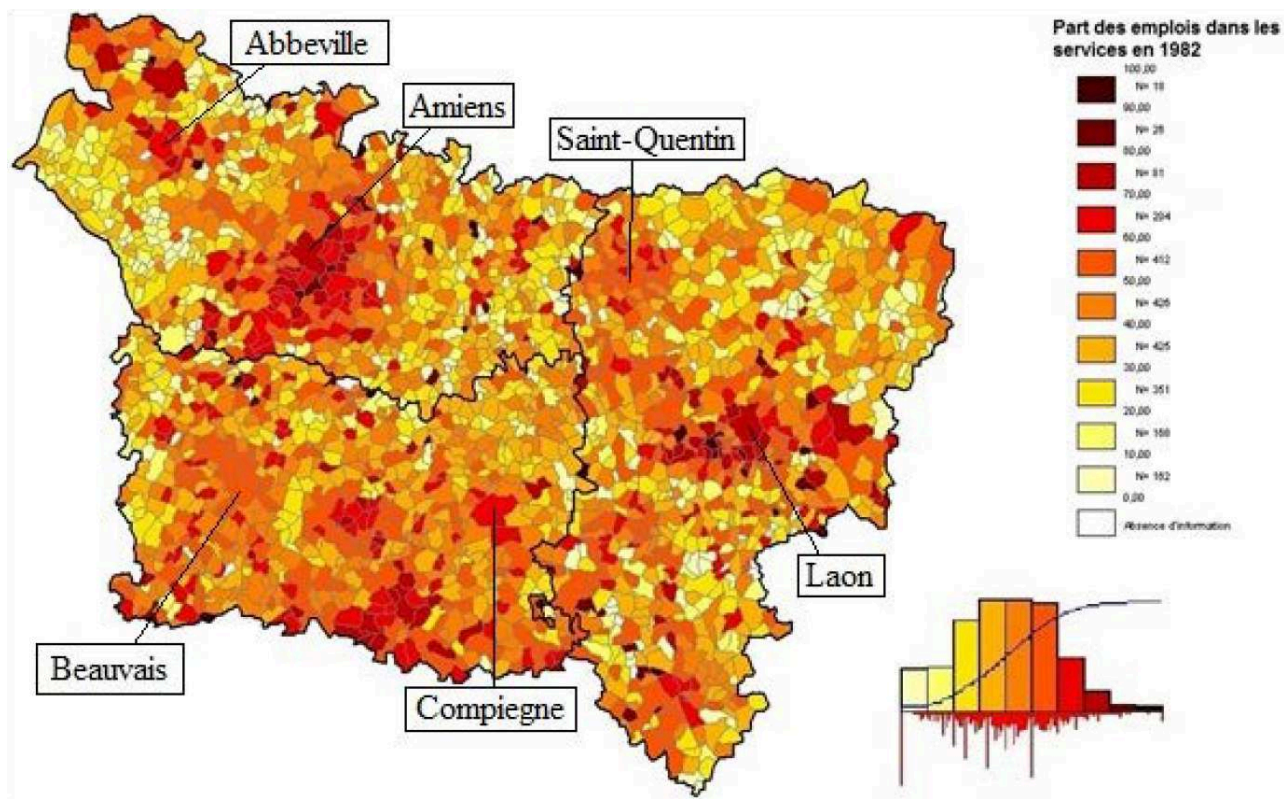

Champs : emplois au lieu de travail dans les services en Picardie Sources : recensements de la population de 1982 
Figure 9. Les communes de Picardie selon la part des emplois dans les services en 2013

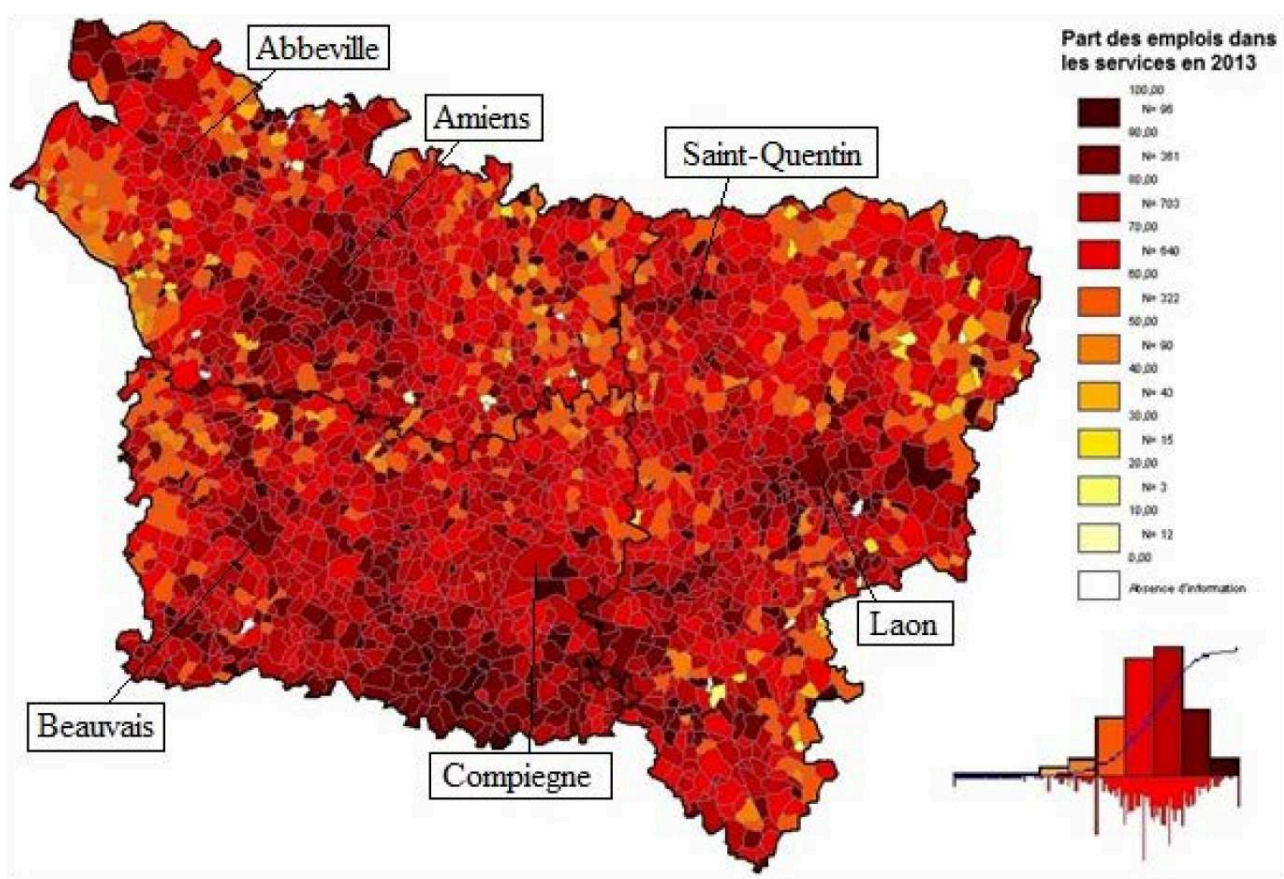

Champs : emplois au lieu de travail dans les services en Picardie

Sources : recensements de la population de 2013 villes et leurs périphéries. Ainsi, Amiens, Laon, et le Sud de l'Oise étaient les espaces où se concentraient les emplois du tertiaire. En 1982, ce secteur représentait au moins $50 \%$ des emplois dans près d'un tiers des communes de la région. En 2013, ce sont $93 \%$ des communes qui dépassent ce seuil. Entre 1982 et 2013, le tertiaire s'est installé dans toute la région, et particulièrement dans l'Oise et le Sud de l'Aisne. Le Nord de l'Aisne a toutefois connu une augmentation du nombre d'emploi dans les services plus nuancée (Figures 8 et 9).

作 nécessairement des phénomènes concomitants : ce n'est pas forcément là où l'industrie a perdu beaucoup d'emplois que les services se sont le plus développés.

L'Oise regroupe la majorité des cantons dans lesquels la baisse de l'emploi industriel a été compensée par l'augmentation du nombre d'emplois dans les services (Montataire et Crépy-en-Valois, par exemple, ont perdu plus de $50 \%$ de leurs emplois industriels, mais le nombre d'emplois dans les services y a plus que doublé). Le département de l'Oise jouit d'une proximité directe avec la région parisienne qui semble favoriser le développement localisé de certaines activités, notamment dans le commerce qui représente $16 \%$ des emplois à Montataire en 2014 contre $12 \%$ en Picardie.

En outre, sur la même période, les cantons urbains ou semi-urbains ont subi de lourdes pertes industrielles sans que le nombre d'emplois dans les services n'y augmente beaucoup. C'est le cas des centres urbains (Amiens, Beauvais, Abbeville, Saint-Quentin, Creil) où les services étaient déjà fortement présents et n'évoluent plus guère à partir des années 2000 (Figure 10). Cette configuration se présente également dans les cantons centrés sur de petites villes éloignées des pôles urbains (comme Hirson, Tergnier, Guise ou Péronne, par exemple) : l'industrie y a massivement perdu des emplois, alors que les

Populations vulnérables, 5 | 2019 
emplois dans les services s'y sont assez peu développés. Il s'agit ici de communes dans lesquelles l'emploi s'appauvrit sous l'effet combiné de la fermeture de sites industriels de production et de la récente tendance à la disparition des services publics de proximité (fermeture de services hospitaliers, d'écoles maternelles et primaires, de bureaux de poste ou encore de gare).

Enfin, les communes les moins industrialisées (et où l'emploi industriel est donc le moins susceptible de baisser), ont été marquées par un fort développement des emplois dans les services. C'est le cas à Ailly-sur-Noye, Saint-Just-en-Chaussée ou La-Fère-enTardenois, par exemple, où l'industrie était peu présente, mais dont la relative proximité avec les centres urbains ou la valorisation de patrimoine ont fortement fait augmenter le nombre d'emplois dans les services.

Figure 10. Les cantons de Picardie selon d'évolution du nombre d'emplois dans les services et dans l'industrie entre 1982 et 2013

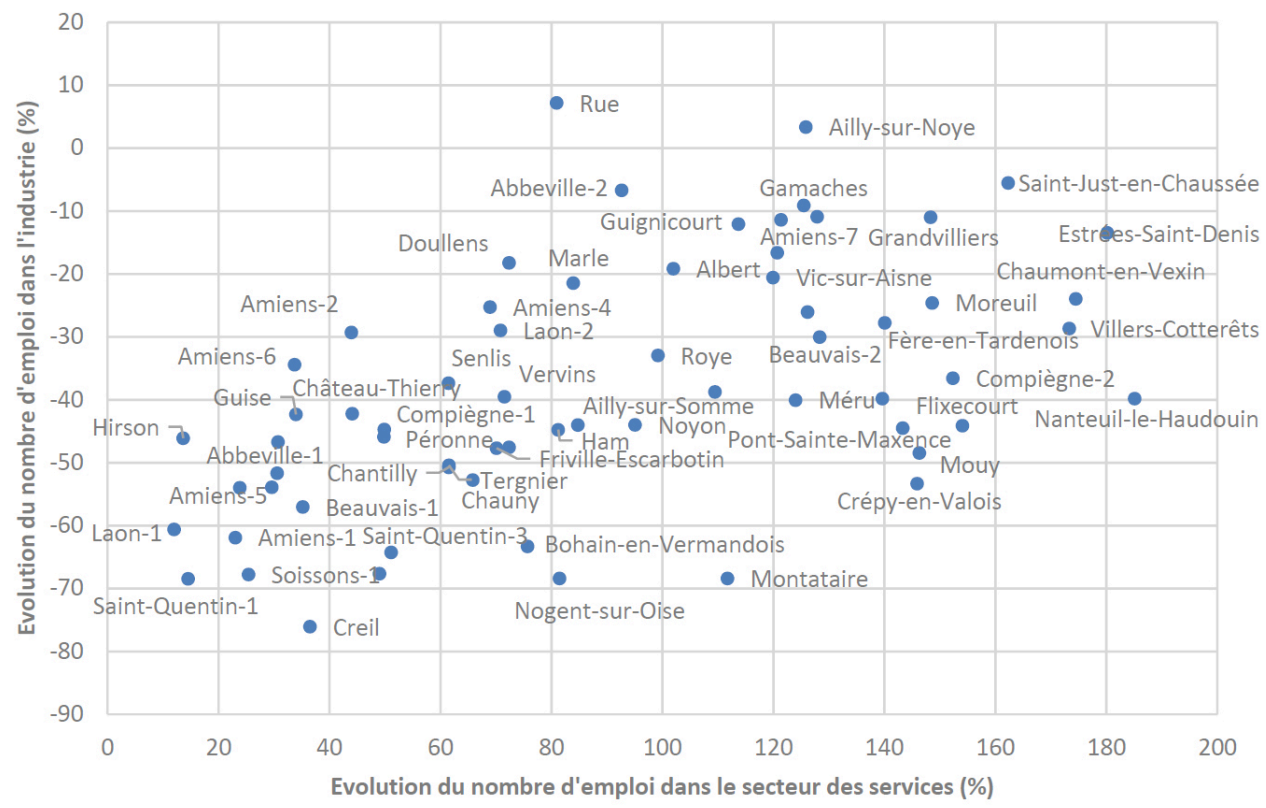

Champs : emplois au lieu de travail des cantons (2014) de Picardie

Sources : recensements de la population de 1982 et 2013

\section{2) Évolution de la morphologie sociale et installation du chômage}

Outre la dynamique du volume et de la structure de l'emploi, la désindustrialisation est une transformation lourde et rapide se caractérisant aussi par l'installation d'un chômage massif. L'évolution de la morphologie sociale des territoires, que l'on peut approcher par l'évolution des parts des PCS dans la population active au lieu de résidence, permet de rendre compte de ce phénomène.

D'une manière générale, la taille de la population active picarde augmente au cours des années 1980 et 1990, puis va entamer une légère baisse, suivant les tendances de l'évolution de l'emploi décrite plus haut. L'augmentation du nombre d'emplois dans les services s'accompagne d'une augmentation du nombre de cadres, de professions intermédiaires et d'employés (Figure 11). Le nombre de cadres en Picardie a plus que doublé entre 1982 et 2013 (augmentation de 107,8 \%, restant inférieure à la moyenne 
nationale qui atteint $130 \%$ ), et c'est dans l'Oise que cette augmentation est la plus importante (124,8\% contre $112 \%$ dans la Somme et $69,2 \%$ dans l'Aisne). C'est également dans l'Oise que se sont le plus accrus les effectifs de personnes employées ou occupant des professions intermédiaires. En 2013, près de $13 \%$ des actifs de ce département sont cadres ou occupent des professions intellectuelles (contre $11 \%$ pour la Picardie), un quart de la population active appartient aux professions intermédiaires ( $23 \%$ en Picardie), et un autre est employé (même proportion que dans la région). L'évolution de la part des ouvriers dans la population active picarde est, quant à elle, résolument orientée à la baisse entre 1982 et 2013, passant de 37,3 \% à 23,1\%.

Figure 11. Évolution (en base 100) du nombre d'actifs résidents en Picardie par PCS entre 1982 et 2013

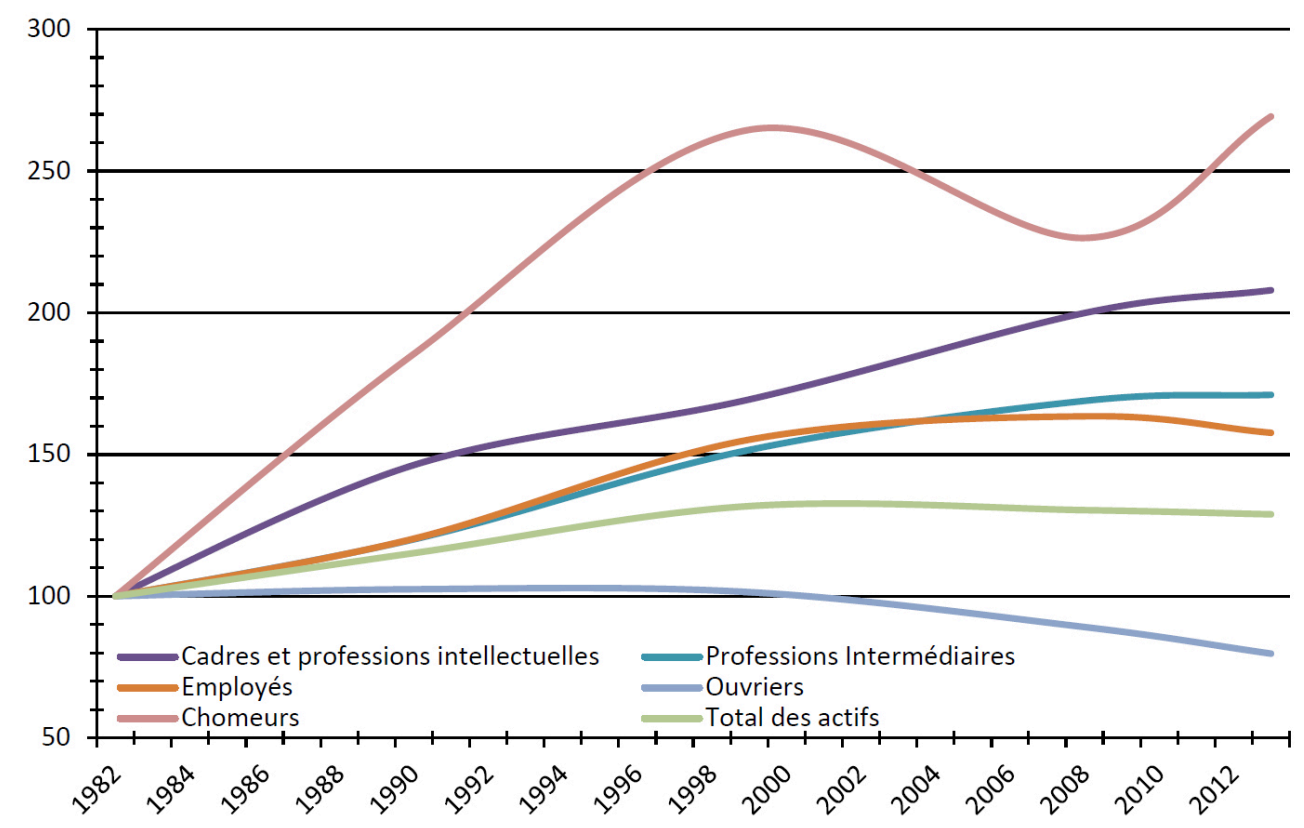

Champs : population active de 25 à 55 ans, France métropolitaine. Pour alléger la lecture du graphique, les PCS Agriculteurs et Artisans-Commerçants ont été retirées

Sources : recensements de la population de 1968, 1975, 1982, 1990, 1999, 2008 et 2013

la morphologie sociale en France, mais plus encore en Picardie, et il existe de fortes disparités entre les départements. Le chômage affecte davantage la Somme (12,6 \% de la population active de 2013$)$ et l'Aisne (14\%) que l'Oise $(10,5 \%$, proche de la moyenne nationale de $10,9 \%)$.

De plus, le chômage a des spécificités territoriales. L'examen des données des inscrits sur les listes de Pôle emploi montre ainsi des différences territorialisées en ce qui concerne les formes de chômage, correspondant aux catégories A, B, C, D et $\mathrm{E}^{4}$, ainsi que les générations touchées (Tableau 2).

mmunes de plus de 5000 habitants de l'Aisne sont caractérisées par une surreprésentation des chômeurs de catégorie A, quelle que soit la classe d'âge considérée. Plus généralement, on peut remarquer une surreprésentation des personnes de 50 ans et plus vivants dans les villes (quel que soit le département) parmi les chômeurs de catégorie A. Il semble par contre que cette catégorie soit moins présente sur les territoires ruraux et villes de moins de 5000 habitants. Dans ces 
espaces, les personnes de 50 ans et plus sont surreprésentées parmi les chômeurs de catégorie B (qui ont exercé une activité réduite au cours du mois). De même, les personnes de 25 à 49 ans en catégorie C (ayant exercé une activité longue) sont fortement présentes dans ces espaces. Le chômage des campagnes semble donc être ponctué d'activités plus ou moins longues, alors que celui des espaces plus urbains se caractérise plutôt par une absence totale d'activité.

Tableau 2. Répartition des chômeurs par catégories selon leur tranche d'âge et leur lieu de résidence

\begin{tabular}{|c|c|c|c|c|c|c|c|}
\hline \multirow[b]{2}{*}{ Localité } & \multirow[b]{2}{*}{ Classe d'âges } & \multicolumn{5}{|c|}{$\begin{array}{l}\text { Catégorie de demande } \\
\text { d'emploi }\end{array}$} & \multirow[t]{2}{*}{ Total } \\
\hline & & A & B & c & D & E & \\
\hline \multirow{3}{*}{$\begin{array}{l}\text { Aisne (communes de plus de } 5000 \\
\text { habitants) }\end{array}$} & $\begin{array}{l}\text { Moins de } 25 \\
\text { ans }\end{array}$ & 61,9 & 7,9 & 13,9 & 11,3 & 5,0 & 100,0 \\
\hline & De 25 à 49 ans & 62,4 & 9,0 & 17,4 & 5,7 & 5,5 & 100,0 \\
\hline & 50 ans et plus & 67,4 & 10,2 & 14,0 & 3,1 & 5,3 & 100,0 \\
\hline \multirow{3}{*}{$\begin{array}{l}\text { Oise (communes de plus de } 5000 \\
\text { habitants) }\end{array}$} & $\begin{array}{l}\text { Moins de } 25 \\
\text { ans }\end{array}$ & 59,0 & 10,7 & 15,3 & 9,5 & 5,5 & 100,0 \\
\hline & De 25 à 49 ans & 57,6 & 10,1 & 21,2 & 6,1 & 5,2 & 100,0 \\
\hline & 50 ans et plus & 64,4 & 10,6 & 16,3 & 3,1 & 5,7 & 100,0 \\
\hline \multirow{3}{*}{$\begin{array}{l}\text { Somme (communes de plus de } 5000 \\
\text { habitants) }\end{array}$} & $\begin{array}{l}\text { Moins de } 25 \\
\text { ans }\end{array}$ & 59,7 & 10,1 & 13,4 & 11,9 & 4,9 & 100,0 \\
\hline & De 25 à 49 ans & 58,9 & 9,6 & 19,5 & 6,0 & 5,9 & 100,0 \\
\hline & 50 ans et plus & 63,7 & 11,6 & 15,7 & 2,5 & 6,6 & 100,0 \\
\hline \multirow{3}{*}{ Communes de moins de 5000 habitants } & $\begin{array}{l}\text { Moins de } 25 \\
\text { ans }\end{array}$ & 52,6 & 11,4 & 21,1 & 9,3 & 5,6 & 100,0 \\
\hline & De 25 à 49 ans & 50,1 & 11,2 & 26,3 & 5,8 & 6,6 & 100,0 \\
\hline & 50 ans et plus & 58,9 & 11,9 & 18,7 & 3,3 & 7,1 & 100,0 \\
\hline \multicolumn{2}{|l|}{ Total Picardie } & 56,4 & 10,7 & 20,9 & 6,0 & 6,1 & 100,0 \\
\hline
\end{tabular}

Champs : inscrits au pôle emploi en mars 2017

Lecture : les cases colorées en rouge distinguent une attraction entre les modalités, les cases en bleu signalent une répulsion. Test Chi2 significatif au seuil de $1 \%$

Source : Pôle emploi

Ce premier examen des variables montre que les transformations de l'emploi et de la morphologie sociale peuvent prendre des formes et des intensités particulières si l'on cherche à dépasser les lectures en termes de moyenne, et à relocaliser le 
questionnement. Les dynamiques diffèrent selon les communes, et un même phénomène (comme le chômage) peut en outre y prendre des formes variables demandant des analyses fines.

\section{Espace de la désindustrialisation et vulnérabilités territoriales}

La désindustrialisation, en s'incarnant à la fois dans la baisse du nombre d'emplois dans l'industrie, dans le développement des activités de services demandant de nouveaux types de qualifications, de savoir-faire et de savoir-être, et dans l'installation du chômage, est un phénomène qui peut prendre des formes différentes selon les territoires. La construction d'une Analyse en composantes principales (ACP) à partir des variables relatives aux transformations de l'emploi (en tenant compte des différents secteurs d'activité) et à la morphologie sociale (en tenant compte de l'importance du chômage) mesurée à l'échelle des cantons de la région nous a permis de dresser un espace de l'ampleur et des formes prises par la désindustrialisation en Picardie.

34 Pour structurer l'espace, nous avons mobilisé huit variables issues des recensements de la population : les évolutions de l'emploi tous secteurs confondus, de l'emploi industriel et de l'emploi dans les services entre 1982 et 2013 ; les parts des cadres et professions intellectuelles, des professions intermédiaires, des employés, des ouvriers et des chômeurs dans la population active de 25 à 55 ans en $2013^{5}$. Au regard des valeurs propres, nous nous intéresserons principalement aux deux premiers axes de l'ACP, qui résument ensemble plus des trois quarts de la variance du nuage de points. Les axes factoriels suivants (résumant moins de $15 \%$ de l'inertie) ont été écartés.

\section{1) L'espace du dynamisme de l'emploi et de la morphologie sociale des cantons de Picardie}

Le premier axe oppose les territoires où se concentrent les catégories sociales aisées (occupant des fonctions de cadres ou professions intermédiaires) aux territoires où sont fortement représentées les classes populaires (ouvriers et chômeurs). Cet axe est également un résumé du dynamisme de l'emploi des cantons. Les espaces où se concentrent les catégories populaires sont également ceux où l'évolution de l'emploi est la plus faible ou négative, alors que les territoires où le nombre d'emplois à le plus augmenté sont principalement habités par des fractions sociales plutôt aisées (Tableau 3).

Le second axe oppose quant à lui les cantons où le nombre d'emplois dans le secteur des services a le plus augmenté, où le nombre d'emplois dans l'industrie a le moins baissé et où la part des ouvriers dans la population active est la plus forte en 2013 aux espaces où l'emploi dans les services s'est peu développé alors que le nombre d'emplois industriels a fortement baissé et où la part des cadres dans la population active de 2013 est la plus élevée. 
Tableau 3. Corrélation des variables actives avec les axes retenus

\begin{tabular}{|l|l|l|}
\hline Variables actives (analyse des rangs) & Axe $\mathbf{1}$ & Axe 2 \\
\hline $\begin{array}{l}\text { Évolution de l'emploi dans le secteur industriel } \\
\text { (variable evindus) }\end{array}$ & 0,3954 & 0,7496 \\
\hline $\begin{array}{l}\text { Évolution de l'emploi dans les services } \\
\text { (variable evserv) }\end{array}$ & 0,4713 & 0,7737 \\
\hline $\begin{array}{l}\text { Évolution de l'emploi tous secteurs } \\
\text { (evemploi) }\end{array}$ & 0,7765 & 0,5694 \\
\hline $\begin{array}{l}\text { Part des cadres et professions intellectuelles dans la population active } \\
\text { (partcpis2013) }\end{array}$ & 0,7663 & $-0,5831$ \\
\hline Part des professions intermédiaires dans la population active (partint2013) & 0,8943 & $-0,3282$ \\
\hline $\begin{array}{l}\text { Part des employés dans la population active } \\
\text { (partemployés2013) }\end{array}$ & $-0,1112$ & $-0,0265$ \\
\hline $\begin{array}{l}\text { Part des ouvriers dans la population active } \\
\text { (partouvriers2013) } \\
\text { (partchomeurs2013) }\end{array}$ & $-0,6454$ & 0,6988 \\
\hline
\end{tabular}


Figure 12. Cercle des corrélations des variables actives avec les axes factoriels

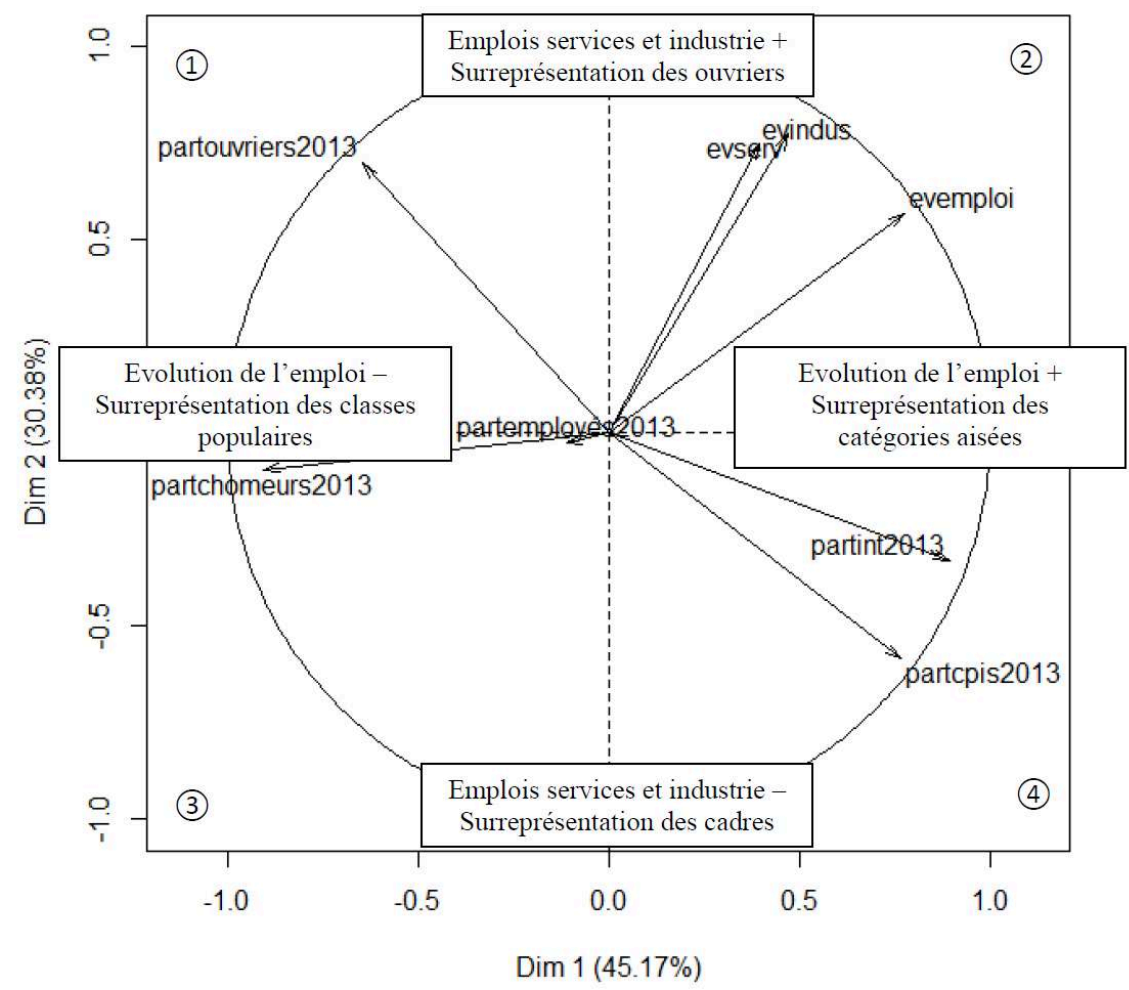

37 Cet espace permet de distinguer les cantons de Picardie en quatre sous-groupes. Le premier (marqué sur les figures 12 et 13 par un (1), en rouge sur la figure 14) regroupe les cantons où les parts d'ouvriers dans la population active locale sont les plus fortes et où la croissance de l'emploi tous secteurs confondus a été la plus faible, voire négative, entre 1982 et 2013. Les cantons regroupés dans cette catégorie (Gamaches, Flixecourt, Marle, Ham, Friville-Escarbotin, Noyon, etc.) sont des zones fortement industrialisées affectées par de lourdes transformations de l'emploi et par l'installation d'un chômage massif. Ces cantons se localisent principalement dans le Nord de l'Aisne et dans la Somme, et il s'agit d'espaces que l'on pourrait dire "en désindustrialisation », au sens où le processus n'est pas terminé. Les pertes sont lourdes, mais l'industrie représentent toujours une part considérable de l'emploi local et les ouvriers constituent une part importante de la population active. 
Figure 13. Projection du nuage des cantons sur le plan factoriel 1 - 2

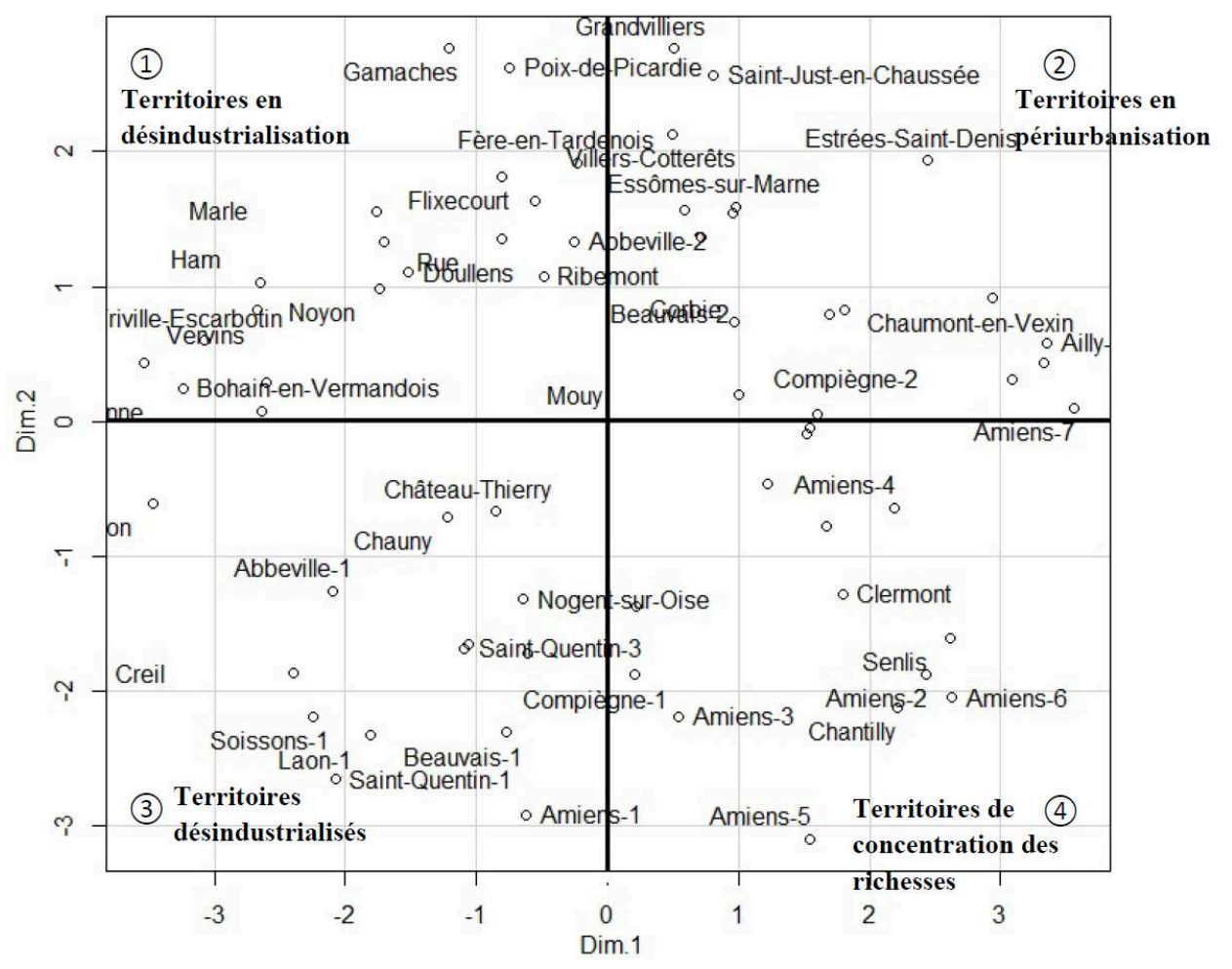

38 À l'opposé du cadran, en diagonale, le sous-groupe (4) concerne les cantons où les parts de cadres, professions intellectuelles et intermédiaires sont les plus élevées. C'est également dans cette catégorie que les parts des personnes au chômage dans la population active sont les plus faibles. Les cantons qui composent cette catégorie (Clermont, Senlis, Chantilly, Amiens 5, 2, 3 et 6) sont les zones où se concentrent les populations aisées et se situent dans le Sud de l'Oise, ou en périphérie d'Amiens, de Laon ou de Compiègne. Ce sont globalement dans ces espaces que se concentrent les richesses.

Les cantons se trouvant dans le cadran (2) sont ceux dans lesquels le nombre d'emplois tous secteurs confondus a le plus augmenté entre 1982 et 2013. Le nombre d'emplois dans les services y a le plus augmenté, et l'industrie y a perdu le moins d'emplois. Ce dynamisme de l'emploi se traduit par de faibles taux de chômage. Ces territoires constituent une large partie de la moitié Sud de la région : l'Oise et le Sud de l'Aisne. Il s'agit d'espaces ruraux faiblement industrialisés en voie de périurbanisation, attirant les travailleurs du secteur tertiaire, salariés dans les pôles urbains ou en région parisienne.

Enfin, la catégorie (3) regroupe les cantons où le nombre d'emplois dans l'industrie a le plus baissé entre 1982 et 2013 et où le nombre d'emplois dans les services a le moins augmenté sur la même période. Ce sont dans ces cantons où les taux de chômage sont les plus élevés. Il s'agit de zones fortement industrialisées où l'activité industrielle a largement décliné depuis les années 1980. On y trouve les cantons de Chauny, Abbeville, Hirson, Saint-Quentin, Creil, Château-Thierry, Soissons, Laon. Ces espaces sont les zones où la désindustrialisation est la plus « aboutie » au sens où l'emploi industriel y a quasiment disparu au cours des quinze dernières années. Le secteur des services s'y développe peu sur la période récente, et le chômage atteint des taux record. 


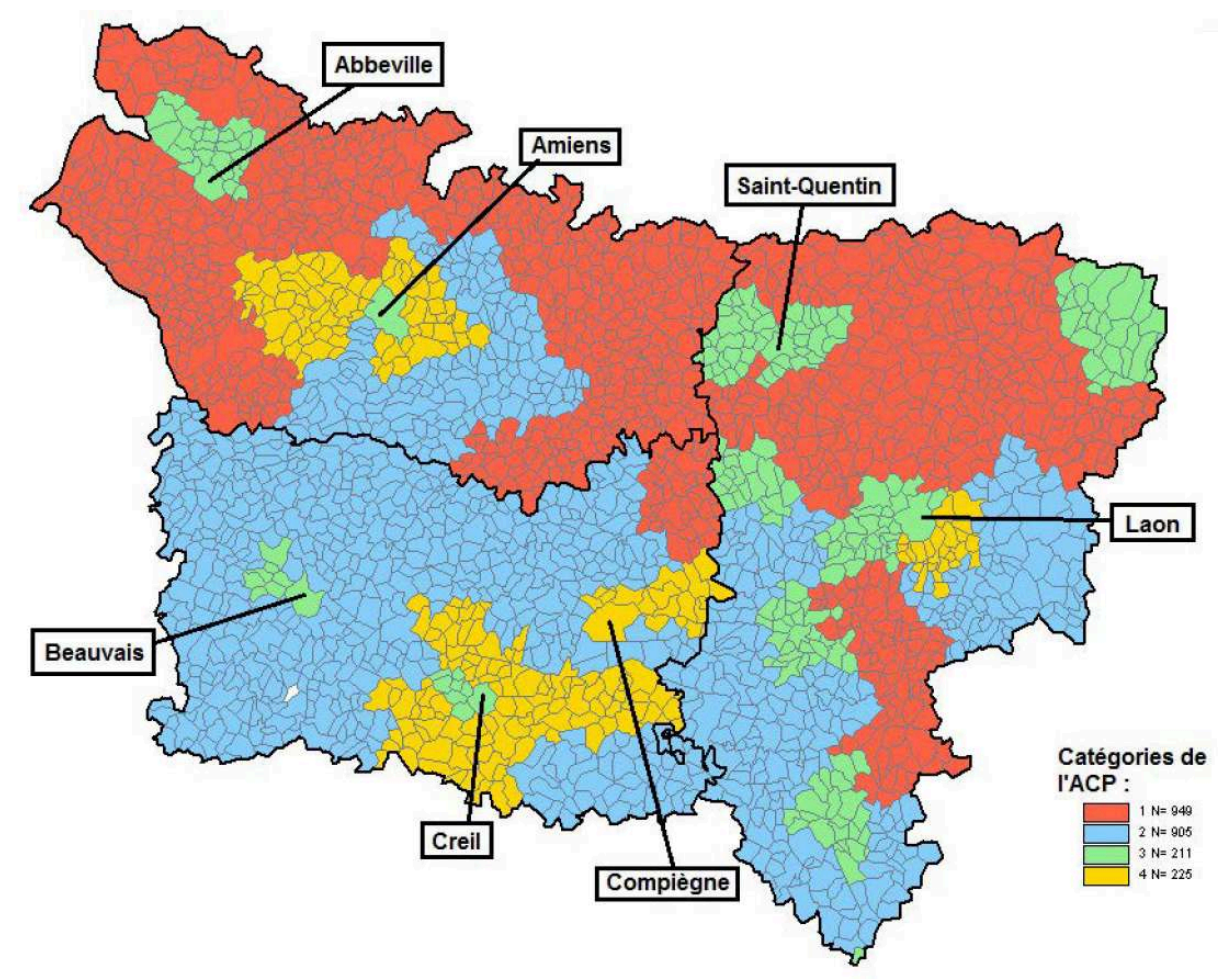

41 À ce stade, nous avons réussi à identifier deux catégories de territoires en Picardie que l'on peut considérer comme désindustrialisés ou en désindustrialisation. Les cantons appartenant aux catégories (1) et (3) constituent les espaces où les pertes d'emplois dans l'industrie n'ont pas été compensées par des gains suffisants dans les services, et où le chômage $\mathrm{a}$ atteint des niveaux plus importants qu'ailleurs.

\section{2) Vulnérabilité sociale et démographique des territoires}

Afin d'enrichir l'analyse, nous pouvons mettre de nouvelles variables en lien avec l'espace factoriel et les catégories de territoire que nous venons d'élaborer, afin de distinguer certains effets concrets de la désindustrialisation sur les populations des différents types de territoires. Nous nous attacherons ici particulièrement à des variables relatives aux revenus et aux niveaux de pauvreté des populations ainsi qu'à leur évolution démographique depuis les années 1980.

\section{Situation de pauvreté et revenus des ménages}

Pour décrire les revenus et les niveaux de pauvreté à l'échelle cantonale, nous avons eu recours aux données du Fichier localisé social et fiscal ${ }^{6}$ de 2013. Plus particulièrement, nous avons retenu les variables relatives à la part des ménages imposables (PartMenImp), au revenu disponible ${ }^{7}$ médian des ménages (RevDispMed_A), à l'indice de Gini calculé sur les revenus des ménages (IndGINI), aux parts des revenus disponibles issues d'activités salariées (SalTrait) et non salariées (RevNonSal), des indemnités chômage (IndCho), ou des prestations sociales (PrestaSoc). 
Les territoires connaissant ou ayant connu la désindustrialisation sont les zones où les revenus des ménages sont les plus faibles et où les parts de ménages imposés sont également les plus basses (Figure 15). Les indemnités chômage et les prestations sociales y représentent une proportion importante des revenus disponibles des ménages, alors que ces espaces ont les plus faibles parts de revenus issues de salaires ou de traitement. Ce sont également sur ces territoires que les inégalités de revenus sont les plus prononcées. Ces caractéristiques témoignent de l'émergence de situations de pauvreté particulières qui apparaissent dans les espaces en désindustrialisation. En comparaison, les « territoires de concentration des richesses » apparaissent comme les zones où les revenus des ménages sont les plus élevés. Ce sont dans ces espaces que la part des revenus d'activités salariées est la plus élevée parmi les revenus disponibles et que les indemnités chômage et les prestations sociales sont assez peu représentées.

Figure 15. Projection des variables de revenu et de pauvreté

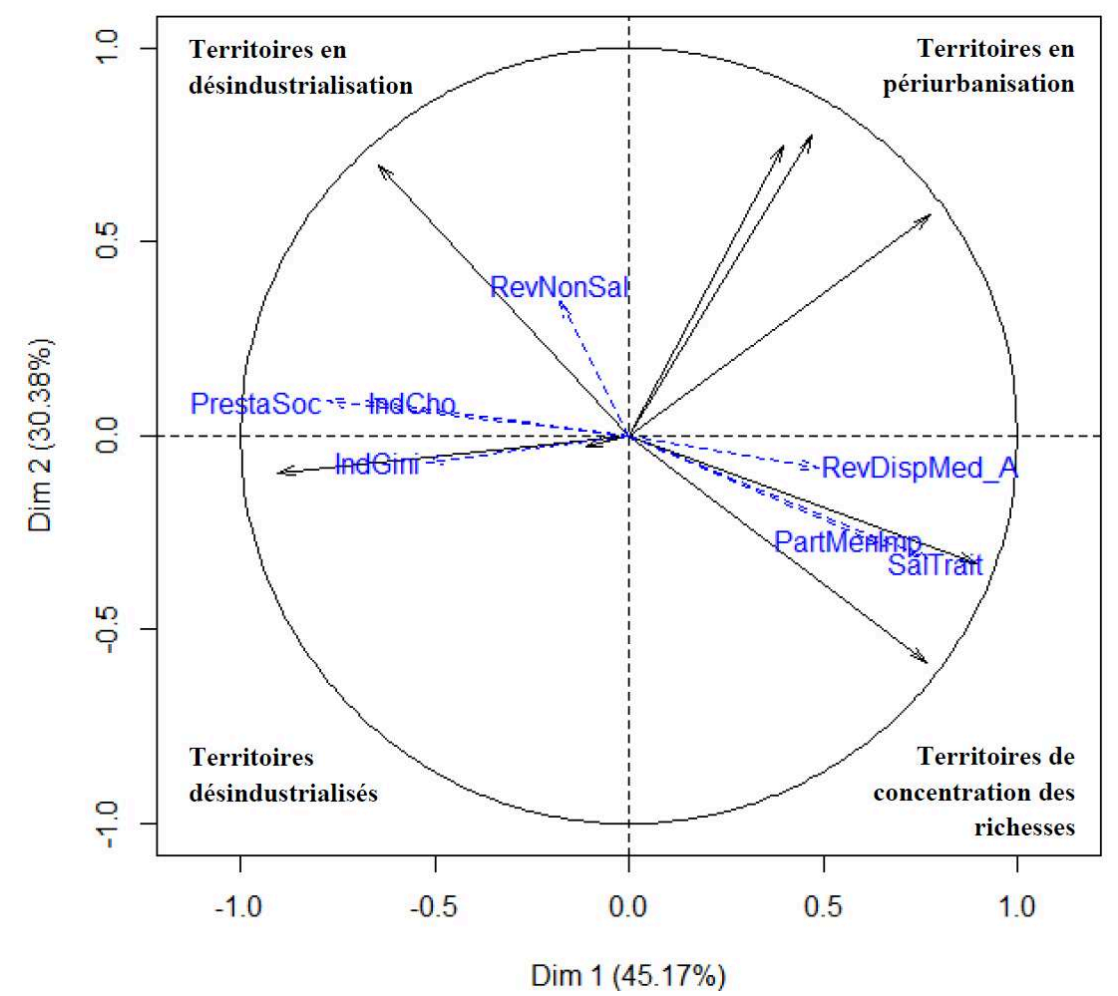

\section{Vieillissement des populations et dépeuplement des espaces}

Enfin, mobilisons des données issues des recensements de 1982 à 2013 afin de caractériser l'évolution de la taille et de la structure des populations résidant dans les cantons de Picardie, afin de mettre en lien ces caractéristiques avec le phénomène de désindustrialisation. Pour cela nous utilisons le taux d'accroissement annuel moyen sur la période 1982-2013 (TxAccAnn) et les évolutions de la population des classes d'âges de moins de 25 ans (EvMoins25ans), de 25 à 50 ans (Ev2550), de 50 à 75 ans (Ev5075ans) et de 75 ans et plus (Ev75ansPlus).

Les territoires en périurbanisation sont ceux dont les taux d'accroissement de la population sont les plus élevés entre 1982 et 2013 et où les classes d'âges les plus jeunes ont gagné en importance (Figure 16). Le dynamisme démographique de ces territoires 
tient au fait qu'ils se peuplent depuis les années 1980 de nouvelles populations, occupant majoritairement des emplois dans les centres urbains. Il s'agit d'une arrivée massive de ménages en âges d'être actifs (25-50 ans), qui sont également les âges où la fécondité est la plus forte, induisant une forte présence des moins de 25 ans.

À l'inverse, les territoires désindustrialisés, ou en désindustrialisation, sont marqués par les plus faibles taux d'accroissement annuel moyen des populations. Les parts des classes d'âges actives ont eu tendance à baisser dans la structure de la population, de même que celles des moins de 25 ans. Ces indicateurs témoignent d'une tendance au dépeuplement des espaces les plus désindustrialisés, compréhensible comme une conséquence du départ massifs des actifs (et notamment des jeunes) pour étudier ou travailler en d'autres endroits. La décroissance relative de ces classes d'âges entraîne une baisse de la fécondité et un vieillissement de la population.

Figure 16. Projection des variables de structure et d'évolution de la population

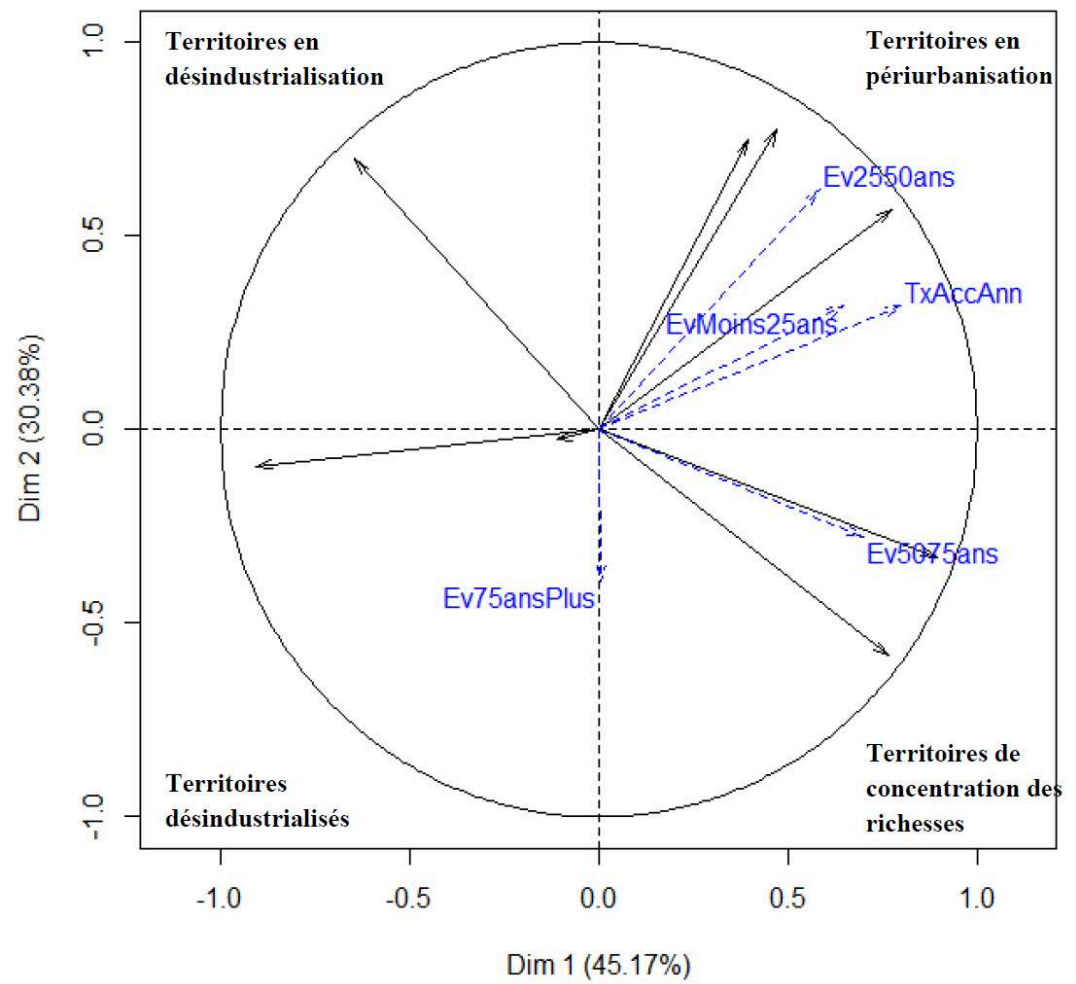

\section{Conclusion}

L'analyse multivariée a permis de décrire les forts écarts entre les territoires de Picardie, illustrant les liens entre les transformations de l'emploi, de la morphologie sociale locale, les niveaux de pauvreté et les dynamiques démographiques. En nous basant sur un petit nombre d'indicateurs et de thématiques, nous avons pu objectiver une partie de l'état des inégalités territoriales au sein de la région, c'est-à-dire mettre en lumière des concentrations spatiales de populations ayant des caractéristiques spécifiques, analysables en termes de vulnérabilités spécifiques. Nous avons pu repérer quatre catégories de cantons pour la Picardie, marqués par des dynamismes différents, parmi lesquels les territoires désindustrialisés ou en désindustrialisation apparaissent 
clairement, marqués notamment par de forts taux de chômage, un haut niveau de pauvreté et un fort dépeuplement et vieillissement de la population.

La désindustrialisation, au-delà des constats de pertes quantitatives d'emplois ou de production, induit l'installation de situations de précarité sociale et économique dans les territoires les plus affectés. Nous avons ainsi pu voir les contours d'une forme de vulnérabilité particulière proprement induite par la désindustrialisation d'espaces fortement ouvriers.

Les territoires désindustrialisés ou en désindustrialisation concentrent les ménages populaires, disposant de faibles revenus dont une grande partie est liée aux prestations sociales et aux indemnités chômage. Le manque de perspectives sur place pousse une part non négligeable des jeunes à quitter le territoire pour suivre une formation ou travailler dans les villes ou zones périurbaines. De même, le manque d'emplois sur place pousse les actifs en recherche d'emploi à envisager différentes formes de mobilités. Par voie de conséquence, la population locale « vieillit par le bas » : les jeunes quittent le territoire et le nombre d'enfants qui y naissent est réduit.

\section{BIBLIOGRAPHIE}

Bacque M-H. et Biewener C. (2013), L'empowerment, un nouveau vocabulaire pour parler de participation?, Idées économiques et sociales, $n^{\circ} 173$, p. 25-32.

Castel R. (1994), « La dynamique des processus de marginalisation : de la vulnérabilité à la désaffiliation », Cahiers de recherche sociologique, $\mathrm{n}^{\circ} 22$, p. 11-27.

Castel R. (1995), Les métamorphoses de la question sociale, Une chronique du salariat, Fayard, Paris.

Castel R. (2009), La montée des incertitudes. Travail, protection, statut de l'individu, Seuil, Paris.

Chauvel L. (2010), Le destin des générations. Structure sociale et cohortes en France du XXe siècle aux années 2010, Paris, PUF.

Collectif du 9 août (2017), Quand ils ont fermél'usine, Lutter contre la délocalisation dans une économie globalisée, Agone, Paris.

Dardot P. et Laval C. (2009), La Nouvelle Raison du monde. Essai sur la société néolibérale, Paris, La Découverte.

Davezies L. (2012), La crise qui vient : la nouvelle fracture territoriale, Paris, Seuil, la République des idées.

Glaymann D. (2005), La vie en intérim, Paris, Fayard.

Laferte G. (2014), « Des études rurales à l'analyse des espaces sociaux localisés. », Sociologie, vol. $5, \mathrm{n}^{\circ} 4$, p. 423-439.

Mendras H. (1988), La Seconde Révolution française, 1965-1984, Paris, Gallimard.

Noiriel G. (2002), Les ouvriers dans la société française, Paris, Seuil.

Roupnel-Fuentes M. (2011), Les chômeurs de Moulinex, Paris, PUF. 


\section{NOTES}

1. Il existe plusieurs méthodes permettant de rendre compte du chômage, chacune reposant sur une définition du phénomène et une méthode de mesure particulière. L'indicateur le plus utilisé est le taux de chômage produit par la Direction de l'animation de la recherche, des études et des statistiques (DARES) du ministère du Travail et calculé à partir des inscriptions à Pôle emploi. Celui-ci cherche essentiellement à donner une vision administrative et politique du phénomène et peut varier indépendamment $d u$ marché du travail (par des modifications des modes d'inscription ou de suivi des demandeurs d'emploi, par exemple). S'il permet d'établir un suivi régulier, mois par mois, de l'évolution du nombre d'inscrits à Pôle emploi, il est contestable sur un plan scientifique. Par ailleurs, le chômage au "sens du Bureau International du Travail», produit par l'INSEE à partir des données issues de l'enquête Emploi, est très efficace en ce qui concerne les comparaisons internationales, mais se base sur un échantillon qui permet assez mal d'élaborer une approche localisée. Nous utiliserons ici le chômage au sens du recensement, c'est à dire dans sa dimension déclarative. Selon le recensement, les chômeurs sont les personnes de plus de 15 ans se déclarant chômeurs (ou ne s'étant déclarés ni en emploi, ni au chômage) et en recherche d'emploi (inscrits ou non à Pôle emploi).

2. "L'effondrement de la population agricole laisse mieux voir combien les mondes ruraux sont aussi historiquement, et aujourd'hui majoritairement, des mondes populaires d'exécution. Cette présence ouvrière s'inscrit dans les transformations industrielles et artisanales dès le XIX $\mathrm{e}^{\mathrm{e}}$ siècle avec la pluriactivité, la proto-industrialisation voire la grande industrie. Une partie de la seconde révolution industrielle et de la mise en usine de la classe ouvrière s'est bien réalisée à la campagne » (Laferté, 2014, p. 130).

3. Lorsque la notion de vulnérabilité est synonyme de difficultés individuelles (risque de pauvreté, exposition à une maladie ou encore situation de handicap), elle attire d'autres notions (anticipation, adaptation, résilience) (Thomas, 2010). Celles-ci nous paraissent problématique car tendant à analyser par les réactions des acteurs des phénomènes plus larges pouvant par exemple se rapporter à des logiques de classes sociales ou de générations (Chauvel, 2010). On voudrait ici éviter les discours inspirés de l'Empowerment pris comme élément d'une rationalité politique considérant les individus comme agissant et prenant des décisions « rationnelles » dans un contexte d'économie de marché généralisé, et devenant des « entrepreneurs de leurs propres vies » (Dardot et Laval, 2009). Ces discours impliquent des capacités individuelles à se positionner rationnellement, à faire des choix, sans toutefois questionner les inégalités sociales, leurs origines et leurs effets. "Tout au plus est évoquée la question de l'accès aux opportunités, sans remise en cause des inégalités sociales » (Bacqué et Biewener, 2013).

4. La catégorie A correspond aux demandeurs d'emploi tenus de faire des actes de recherche d'emploi et sans emploi; la catégorie B regroupe les demandeurs d'emploi tenus de faire des actes de recherche d'emploi et ayant exercé une activité 78 heures ou moins au cours du mois ; la catégorie $\mathrm{C}$ concerne les demandeurs d'emploi tenus de faire des actes de recherche d'emploi et ayant exercé une activité de plus de 78 heures au cours du mois ; la catégorie D : les demandeurs d'emploi non tenus de faire des actes de recherche d'emploi en raison d'un stage, d'une formation ou d'une maladie ; la catégorie E regroupe les demandeurs d'emploi non tenus de faire des actes de recherche d'emploi, en emploi (par exemple : bénéficiaires de contrats aidés).

5. Pour éviter les déformations de l'espace liées à des valeurs extrêmes, nous avons effectué l'ACP sur les rangs plutôt que sur les valeurs. On utilise alors de Rho de Spearman pour déterminer les corrélations entre les variables rangées. L'ACP a été réalisée avec le logiciel R.

6. Construit par l'INSEE, ce fichier regroupe les données fiscales issues de la Direction générale des finances publiques (déclarations de revenus des personnes physiques, taxe d'habitation et fichier d'imposition des personnes physiques) et des données sur les prestations sociales en 
provenance de la Caisse nationale des allocations familiales, de la Caisse nationale d'assurance vieillesse et de la Caisse centrale de la mutualité sociale agricole.

7. Le revenu disponible d'un ménage comprend les revenus d'activité (nets des cotisations sociales), revenus du patrimoine, transferts en provenance d'autres ménages et prestations sociales (y compris les pensions de retraite et les indemnités de chômage), nets des impôts directs.

\section{RÉSUMÉS}

La désindustrialisation et les fermetures de sites de production industrielle sont principalement analysés par le biais des mutations des appareils productifs, ou par leurs effets sur les trajectoires individuelles des travailleur'se's de l'industrie. Nous proposons dans cet article de questionner ces phénomènes en tant que facteur de vulnérabilité des populations et des territoires pris dans leur ensemble.

En nous centrant sur la Picardie, qui fut l'une des régions les plus industrielles de France, nous chercherons à caractériser la désindustrialisation des espaces qui la compose en mobilisant des données issues de différentes sources. Nous mettrons ensuite ces indices de la désindustrialisation en lien avec des indicateurs de pauvreté et de dynamisme démographique afin de tenter une première caractérisation de la vulnérabilité des territoires.

\section{INDEX}

Mots-clés : vulnérabilité, territoires, morphologie sociale, désindustrialisation, Picardie

Keywords : vulnerability, territories, social morphology, deindustrialization, Picardie

\section{AUTEUR}

\section{THOMAS VENET}

Université de Picardie Jules Verne, Centre amiénois de recherche sur l'éducation et la formationEA 4697 